ISSN 1112-9867

\title{
EFFECTS OF LUTEOLIN ON INTRA PERITONEAL ADHESION BANDS IN RAT
}

A. Mahmoud Hashemzaei ${ }^{1}$, B. Ameneh Badrooze ${ }^{1}$, C. Kaveh Tabrizian ${ }^{1}$, D. Mehrdad Iranshahi ${ }^{2}$, E. Mehdi Khoshsima Shahraki ${ }^{3}$, F. Esmaeil Azimi Khatibani and G. Jafar Shahraki ${ }^{1{ }^{1 *}}$

${ }^{1}$ Department of Pharmacology and Toxicology, Faculty of Pharmacy, Zabol University of Medical Sciences, Zabol, Iran

${ }^{2}$ Biotechnology Research Center and School of Pharmacy, Mashhad University of Medical Sciences, Mashhad, Iran

${ }^{3}$ Department of pathology, Faculty of medicine, Zabol University of Medical Sciences, Zabol, Iran

Published online: 05 June 2016

\begin{abstract}
Intraperitoneal adhesion is consequences of surgery in the abdomen and result in infertility, intestinal obstruction and pelvic pain. Luteolin (LUT) is a natural product that has antiinflammatory, anti-oxidant, anti-angiogenic effects and its effects were evaluated as anti-adhesive in animal model. 30 male rats assigned in to 5 groups (6 animals in each group) of LUT 10, 25 and $50 \mathrm{mg} / \mathrm{kg}$ and normal saline as a control group and intact animals. Animal anesthetized and peritoneal injury was conducted and seven days following to surgery, they scarified. Microscopic, macroscopic, lipid peroxidation changes and TNF- $\alpha$ plasma concentration were evaluated. LUT reduced microscopic and macroscopic injury and lipid peroxidation significantly. Plasma concentrations of TNF- $\alpha$ didn't show any changes. LUT reduces lipid peroxidation, adhesion band formation in microscopic and macroscopic scale but no change in the serum levels of TNFalpha.
\end{abstract}

Keywords: Luteolin; Adhesion Bands; TNF- $\alpha$; Lipid Peroxidation.

Author Correspondence, e-mail: jafar.shahraki@gmail.com

doi: http://dx.doi.org/10.4314/jfas.8vi2s.1 


\section{INTRODUCTION}

The consequences of abdominal adhesion that occur after surgery are intestinal obstruction, primary and secondary infertility, pelvic pain and increasing the burden of healthcare resources [1]. The mechanism of intraabdominal adhesion still is to be elucidated but after injury of peritoneum, mesothelium and endothelium activate [2]. Following the activation, inflammatory cytokines including tumor necrosis factor (TNF- $\alpha$ ) and other cytokines release into the peritoneum cavity [2-4]. TNF- $\alpha$ which is produced via activated macrophage is a cytokine that increase in the early phase of wound healing [5]. Then neutrophils, macrophages and eosinophils recruit in the location and start to release fibrinous exudates [2]. In addition, stress oxidative and lipid peroxidation starts and leads to formation of nascent exudates [2]. Until now many drugs and natural products were used to decrease adhesion formation but a satisfactory approach to this problem has yet to be found. The products used for this purpose not enough good to reduce the formation of adhesion or problem in using in the place or their effects are not good $[6,7]$.

Natural products are famous for their anti-oxidant, anti-inflammatory and protective effects on multiple organs $[8,9]$. One of them is Luteolin $(3,4,5,7$-tetra-hydroxylflavone) (LUT) that is found in celery, green pepper, perilla leaf and seed, chamomile tea, and Lonicera japonica [10]. It poses divers effects including anti-cancer, anti-oxidant, anti-inflammatory, cardioprotective and neuroprotective effects. It is a potent inhibitor of lipopolycachasid-induced TNF- $\alpha$ and IL-6 production, as well as nitric oxide expression. It has been elucidated that LUT attract researchers' attraction for its effects and many investigations were done in it. Histopathological examination indicated that Lut alleviated infiltration of inflammatory cells and synovial hyperplasia as well as protected joint destruction in animal model[11]. Furthermore, it can potentiate pain killing effects by inhibition of COX enzymes especially COX 2 and due to anti-inflammatory effects $[12,13]$. As the literature survey shows that Lut may pose anti-inflammatory activity, therefore the objective of the present study was to determine the efficacy of Lut in the post-operative intraperitoneal band formation.

\section{MATERIAL AND METHOD}

\subsection{Materials}

LUT were synthesized by Iranshahi in the Mashhad University of Medical Sciences. TNF- $\alpha$ kit were purchased from RayBiotech Co [14]. 


\subsection{Animals}

A total 30 male Wistar rats between 9 to 10 weeks age (about 200-250 g) obtained from Zabol University of Medical Sciences, were used in this study. The animals were housed in the normal condition (free access to food and water, $12 \mathrm{~h} / 12 \mathrm{~h}$ dark, light circadian rhythm). All experimental procedure was under Helsinki guidance and was accepted by ethics committee of Zabol University of Medical Sciences.

\subsection{Surgical procedure}

Animals were anesthetized, using an intraperitoneal [15] injection of ketamine $100 \mathrm{mg} / \mathrm{kg}$ and xylazine $10 \mathrm{mg} / \mathrm{kg}$. After shaving and preparation of the site of surgery with alcohol and iodine solution, $3 \mathrm{~cm}$ laparotomy was performed to gain access to the abdominal cavity. Using technique of Peritoneal Button Creation (PBC), which is considered the most consistent and reproducible technique, induction of adhesion performed [14]. For formation of four peritoneal buttons barbed 2.0 polypropylene sutures in a chain alignment were used. Each suture encapsulated approximately $2 \mathrm{~cm}$ of parietal peritoneum; the diameter of the tied sutures were approximately $5 \mathrm{~mm}$. LUT in concentrations 10,25 and $50 \mathrm{mg} / \mathrm{kg}$ were injected peritoneal and then the peritoneum was sutured. They were kept in the recovery room for five days. After 7 days, animals were sacrificed after they anesthetized using ketamine $100 \mathrm{mg} / \mathrm{kg}$ and xylazine 10 $\mathrm{mg} / \mathrm{kg}$. Then, the rats underwent a laparotomy and peritoneal samples were sent for a histopathology evaluation and adhesion formation intensity was recorded based on Adhesion Scoring System (ASS). Furthermore, peritoneal samples harvested for lipid peroxidation and histopathological examinations. Blood samples were obtained to determine plasma TNF- $\alpha$ concentration.

\subsection{Statistics}

The results were expressed as mean $\pm \mathrm{SD}$. One-way ANOVA and $\mathrm{T}$ tests were performed, using SPSS version 16 (SPSS Inc., Chicago, Illinois, United States). The Kruskal-Wallis test was performed to identify the differences in the adhesion grades among the groups. P-values less than 0.05 were accepted as significant.

\section{RESULTS}

\subsection{Measurement of macroscopic adhesion degree}

Macroscopic adhesion divided in to 5 grades based on Yilmaz HG et al [16] as follow: Grade 0; No fibrosis grade 1; thin bunches of a cellular fibrosis grade 2; wide areas of fibrosis with 
reduced vascularization grade 3; areas of fibrosis formed by thick bunch of collagen, 4; two areas of fibrosis and 5; more than two areas of fibrosis (figure 1). The results showed significant reduction in the severity of adhesion formation in LUT groups in comparison to NS groups $(\mathrm{p}<0.05)$.

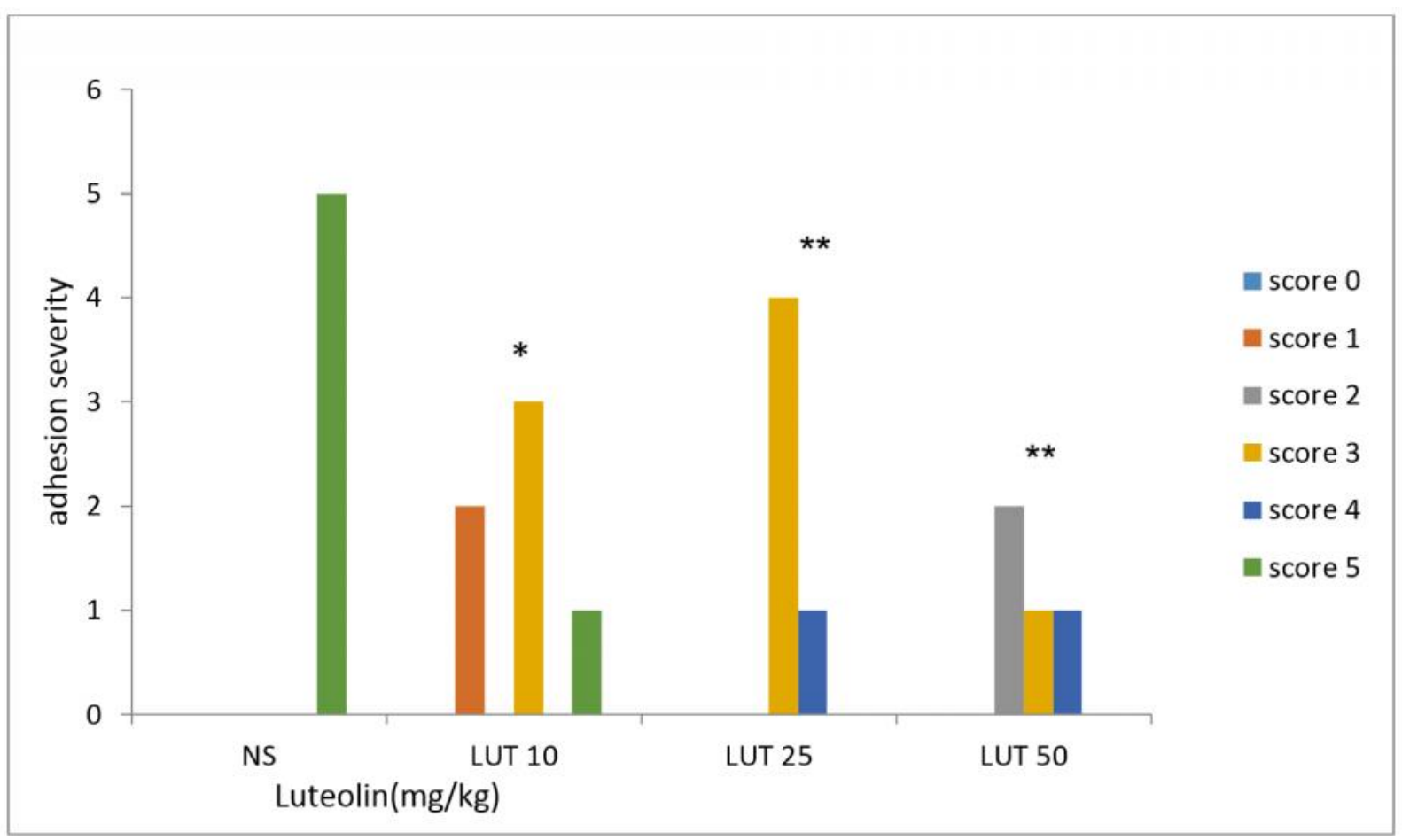

Fig.1. Comparison of the effects of LUT on peritoneal macroscopic adhesions in different groups of Wistar rats. Numbers of rats based on adhesion severity scores in LUT $(10,25,50 \mathrm{and} \mathrm{mg} / \mathrm{kg})$ and control [15] groups are shown $(* P<0.05$ and $* * P<0.01$ was considered significant for each group)

\subsection{Measurement of microscopic adhesion degree}

In the evaluation of microscopic adhesion, pathologist checked adhesion with respect to inflammation, fibrosis and neovascularization (Fig.2). Based on Y1lmaz et al [16] microscopic scales divided into four scores of, 1; fibrin aggregation and neutrophil infiltration, score 2; edema, granulation, infiltration and migration of connective tissue, score 3 ; formation of collagen and score 4; formation of fibrous. Microscopic adhesion in the treatment groups were significantly decreases in comparison to NS group $(\mathrm{p}<0.05)$. 


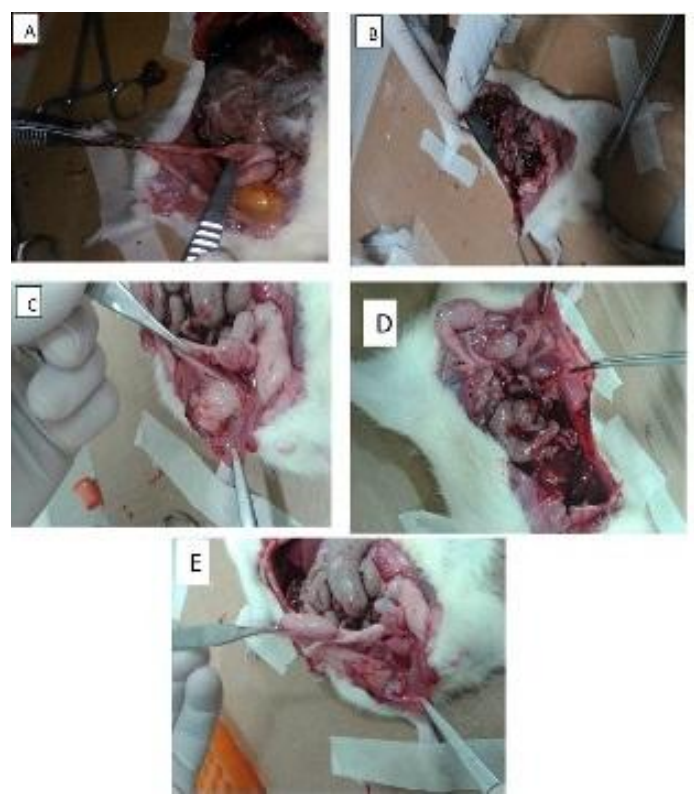

Fig.2. Adhesion band scores based on ASS method: A: score 1, single, filmy adhesion, B: score 2, more than filmy adhesion; C: score 3, single, dense adhesion band; D \& E: score 4 single, dense adhesion band with surface adhesion and score 5, more than one dense adhesion band; A and B are related to LUT and C, D \& E are related to the NS group

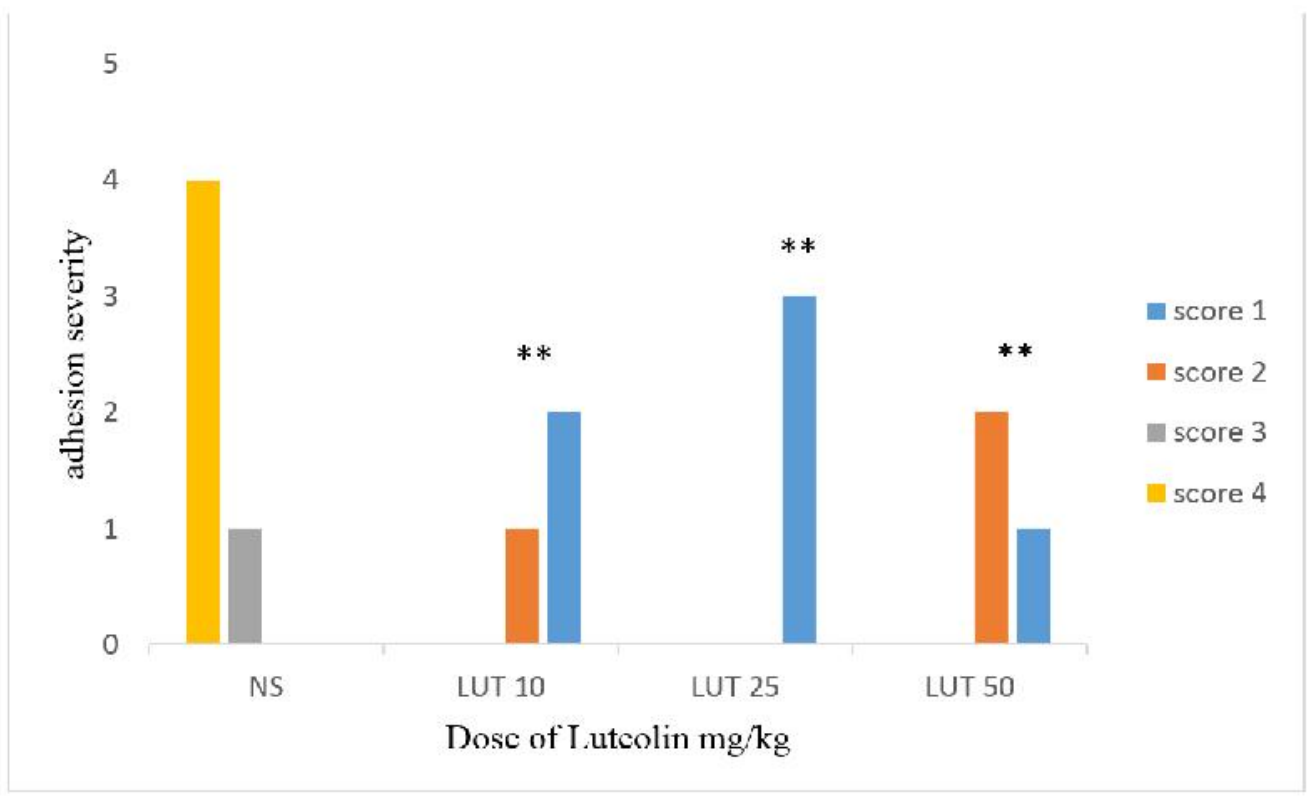

Fig.3. Comparison of the effects of LUT on peritoneal microscopic adhesions in different groups of Wistar rats. Numbers of rats based on microscopic findings in LUT (1o, 25 and $50 \mathrm{mg} / \mathrm{ml})$ and control (NS) groups are shown in the figure $(* * \mathrm{P}<0.01$ and $* \mathrm{P}<0.05$ was considered significant $)$ 


\subsection{Measurement of lipid peroxidation}

Results of lipid peroxidation showed that LUT in three doses (10, 25 and $50 \mathrm{mg} / \mathrm{kg}$ ) decreased lipid peroxidation in comparison to NS $(* * * \mathrm{p}<0.001$ between groups in comparison to intact animals, \#\#\# p<0.001 in comparison to NS group).

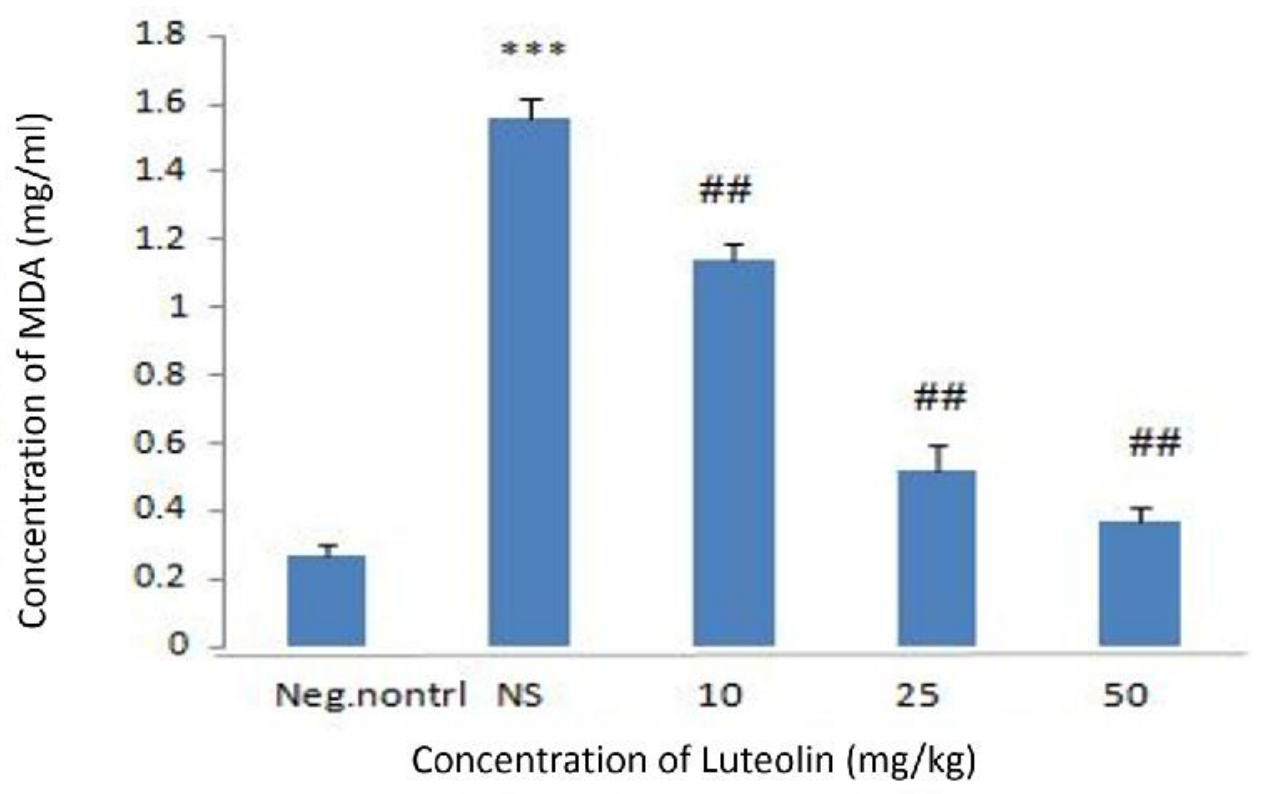

Fig.4. comparison of the effects of Luteolin $(10,25$ and $50 \mathrm{mg} / \mathrm{kg}$ ) on lipid peroxidation in the rats. (*** showed $p<0.001$ in comparison to negative control, \#\# showed $p<0.01$ in comparison to normal saline)

\subsection{Elisa assay of TNF- $\alpha$ in blood}

There were not showed any significant differences between treatment and control groups. The blood samples collected from animals after two weeks following the beginning of procedure. 


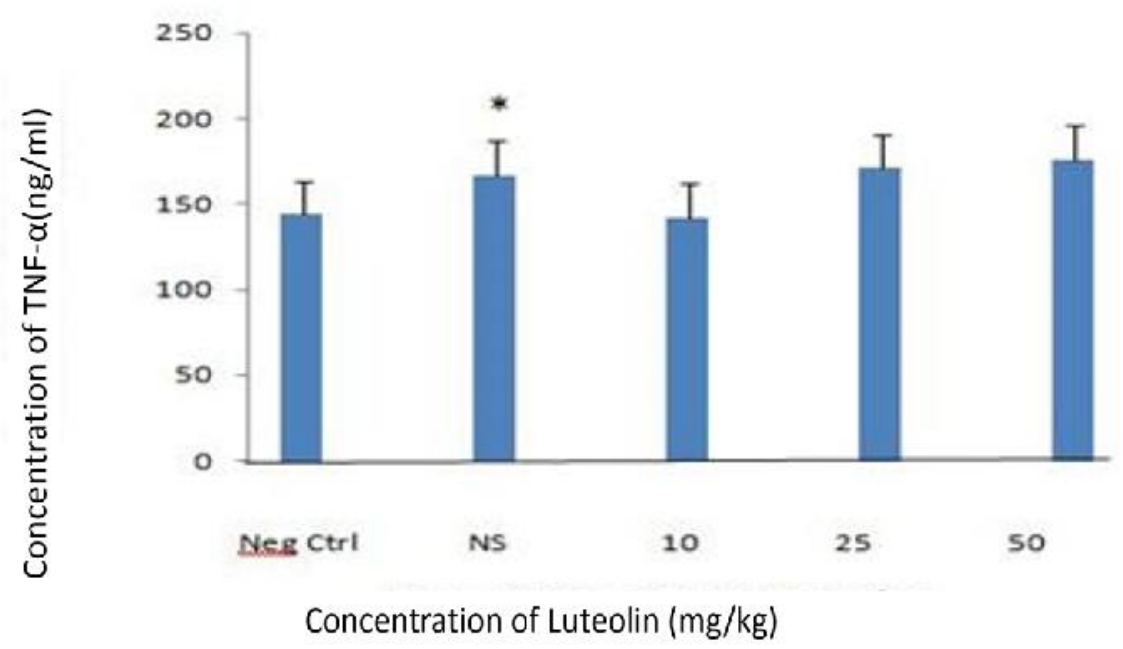

Fig.5. Comparison of changes in TNF- $\alpha$ serum concentrations in different groups of LUT $(10,25$ and $50 \mathrm{mg} / \mathrm{kg}$ ) in comparison to control group (NS) of Wistar rats. In the intact group in comparison to NS group, changes was significant $(* \mathrm{P}<0.05)$ but LUT didn't reduce the amount of plasma TNF- $\alpha$ levels $(\mathrm{P}>0.05)$

\section{DISCUSSION}

Any kind of abdominal surgery result in adhesion and clinical, economical and legal outcome. Up to know there is no cure for it and prevention of adhesion formation is one of the greatest demand of surgeons worldwide. Unlike using many compounds including natural products and drugs with the properties of anti-inflammatory, anti-oxidant, COX inhibitors and anti-adhesion there were not showed any advances in prevention of adhesion formation $[6,7,13,17]$. In this study the effects of LUT on prevention of post-operative adhesion formation was evaluated. The results showed that LUT $(10,25$ and $50 \mathrm{mg} / \mathrm{kg}$ ) decreased adhesion formation in microscopic and macroscopic scale. Furthermore, there are decrement in lipid peroxidation in the treatment groups in comparison to NS group and there were not shown any significant decreasing in blood levels of TNF- $\alpha$ in treatment groups in comparison to NS.

Infiltration of fibrinous exudates outside of vessel is the consequences of vessel dilation following to peritoneal mesothelium cells injury that is made of fibroblasts and extracellular matrix that leads to sediment scar formation [4]. Moreover, aggregation of macrophages in the 
location can be potentiated by inflammatory factors including plasminogen, TNF- $\alpha$, IL-1, IL-6 and [18] which led to the adhesion of peritoneal site.

LUT has divers beneficial effects including anti-cancer, anti-oxidant, anti-inflammatory, cardioprotective and neuroprotective [12]. Although the anti-adhesive mechanisms of LUT are not completely understood, anti-angiogenic and anti-inflammatory effects are possibly the main reasons for LUT effects. Immunosuppressants, corticosteroids and COX-2 inhibitors can reduce post-operative adhesion formation in animal models $[2,13,19]$. One of the mechanism that LUT can affects is COX inhibition. COX-2 is an inducible enzyme that inhibitors of this enzyme reduce adhesion formation $[13,20]$. Our study is in consistent with them in a way that it can inhibit iNOS expression and COX-2 activity [12, 21]. Furthermore, antagonist of TNF-alpha can diminish adhesion formation in animals' models [19]. Past studies confirmed that LUT can inhibit production of pro inflammatory cytokines including TNF-alpha, IL-1 and IL-6.

LUT can inhibit activity of COX-2 and as a consequence, PGE2 production. PGE2 pose strong inflammatory effects in vitro as well as increasing COX-2 expression and iNOS activation [21, 22]. Inhibition of COX-2 result in PGE2 decrement and it is likely one of the mechanism that LUT can decrease band formation following to adhesion induction in animal model. Past studies confirmed that COX inhibitors can alleviate post-operative adhesion formation in animal model. Altogether, the current study showed that LUT decreased adhesive bands but did not reduce serum level of TNF- $\alpha$ in the treated group. Explanation for the observed results might include a) given the local administration of LUT (instead of its systemic application), local content (peritoneal exudates content) level of biomarkers need to be evaluated. If LUT were administered intravenously, serum levels of TNF- $\alpha$ might have significantly changed. Previous study was done by cheong et al showed that peritoneal exudate concentrations of cytokines are different from their serum levels [3]. They showed that adhesion formation within $12 \mathrm{~h}$ after surgery was correlated with IL-1 and IL-6 intra-peritoneal concentration [3]; b) other adhesive, angiogenic and pro-inflammatory factors in the formation of postoperative adhesion bands may have a role; c) significant changes in the serum levels of biomarkers depends on the pathophysiology of adhesion bands due to the involvement of many factors in their formation. 


\section{CONCLUSION}

In the present study, it was confirmed that LUT decreased the formation of postoperative adhesive bands at microscopic and macroscopic levels as well as lipid peroxidation but there were not showed any significant differences between plasma levels of TNF- $\alpha$.

\section{ACKNOWLEDGMENT}

The authors thank the Vice Chancellor of Research, Zabol University of Medical Sciences for financial support.

\section{REFERENCES}

[1] Ellis H, The clinical significance of adhesions: focus on intestinal obstruction. The European journal of surgery Supplement:= Acta chirurgica Supplement. 1996, (577):5-9

[2] Maciver AH, McCall M, Shapiro AJ, Intra-abdominal adhesions: cellular mechanisms and strategies for prevention. International Journal of Surgery. 2011, 9(8):589-94

[3] Cheong Y, Laird S, Shelton J, Ledger W, Li T, Cooke I, The correlation of adhesions and peritoneal fluid cytokine concentrations: a pilot study. Human Reproduction. 2002, 17(4):103945

[4] Eckes B, Nischt R, Krieg T, Cell-matrix interactions in dermal repair and scarring. Fibrogenesis \& tissue repair. 2010, 3(1):4

[5] Holmdahl L, Ivarsson ML, The role of cytokines, coagulation, and fibrinolysis in peritoneal tissue repair. European Journal of Surgery. 1999, 165(11):1012-9

[6] Jomezadeh V, Mohammadpour AH, Rajabi O, Tavassoli A, Maddah G, Evaluation of curcumin effects on post-operative peritoneal adhesion in rats. Iranian journal of basic medical sciences. 2012, 15(6):1162

[7] Mohammadpour AH, Tavassoli A, Khakzad MR, Zibaee E, Afshar M, Hashemzaei M, Karimi G, Effect of gold nanoparticles on postoperative peritoneal adhesions in rats. Nanomedicine Journal. 2015, 2(3):211-6

[8] Heim KE, Tagliaferro AR, Bobilya DJ, Flavonoid antioxidants: chemistry, metabolism and structure-activity relationships. The Journal of nutritional biochemistry. 2002, 13(10):572-84

[9] Lin Y, Shi R, Wang X, Shen H-M, Luteolin, a flavonoid with potentials for cancer prevention and therapy. Current cancer drug targets. 2008, 8(7):634 
[10] Tsai F-S, Peng W-H, Wang W-H, Wu C-R, Hsieh C-C, Lin Y-T, Feng I-C, Hsieh M-T, Effects of luteolin on learning acquisition in rats: involvement of the central cholinergic system. Life sciences. 2007, 80(18):1692-8

[11] Shi F, Zhou D, Ji Z, Xu Z, Yang H, Anti-arthritic activity of luteolin in Freund's complete adjuvant-induced arthritis in rats by suppressing P2X4 pathway. Chemico-biological interactions. 2015, 226:82-7

[12] $\mathrm{Hu}$ C, Kitts DD, Luteolin and luteolin-7-O-glucoside from dandelion flower suppress iNOS and COX-2 in RAW264. 7 cells. Molecular and cellular biochemistry. 2004, 265(1-2):107-13

[13] Greene AK, Alwayn IP, Nose V, Flynn E, Sampson D, Zurakowski D, Folkman J, Puder M, Prevention of intra-abdominal adhesions using the antiangiogenic COX-2 inhibitor celecoxib. Annals of surgery. 2005, 242(1):140

[14] Whang SH, Astudillo JA, Sporn E, Bachman SL, Miedema BW, Davis W, Thaler K, In search of the best peritoneal adhesion model: comparison of different techniques in a rat model. Journal of Surgical Research. 2011, 167(2):245-50

[15] Heydrick SJ, Reed KL, Cohen PA, Aarons CB, Gower AC, Becker JM, Stucchi AF, Intraperitoneal administration of methylene blue attenuates oxidative stress, increases peritoneal fibrinolysis, and inhibits intraabdominal adhesion formation. Journal of Surgical Research. 2007, 143(2):311-9

[16] Yilmaz HG, Tacyildiz IH, Keles C, Gedik E, Kilinc N, Micronized purified flavonoid fraction may prevent formation of intraperitoneal adhesions in rats. Fertility and sterility. 2005, 84:1083-8

[17] Chu DI, Lim R, Heydrick S, Gainsbury ML, Abdou R, D’Addese L, Reed KL, Stucchi AF, Becker JM, N-acetyl-l-cysteine decreases intra-abdominal adhesion formation through the upregulation of peritoneal fibrinolytic activity and antioxidant defenses. Surgery. 2011, 149(6):801-12

[18] Vykoukal D, Davies MG, Vascular biology of metabolic syndrome. Journal of vascular surgery. 2011, 54(3):819-31

[19] Kurukahvecioglu O, Koksal H, Gulbahar O, Erdem O, Engin D, Yazicioglu O, Kerem M, Taneri F, Infliximab" TNF-alpha antagonist" decreases intraabdominal adhesions. Saudi medical journal. 2007, 28(12):1830-5 
[20] Greene AK, Puder M, Prevention of intra-abdominal adhesions using the antiangiogenic COX-2 inhibitor celecoxib. Annals of surgery. 2006, 244(2):328

[21] Harris GK, Qian Y, Leonard SS, Sbarra DC, Shi X, Luteolin and chrysin differentially inhibit cyclooxygenase-2 expression and scavenge reactive oxygen species but similarly inhibit prostaglandin-E2 formation in RAW 264.7 cells. The Journal of nutrition. 2006, 136(6):1517-21 [22] O'Leary KA, Pascual-Tereasa Sd, Needs PW, Bao Y-P, O’Brien NM, Williamson G, Effect of flavonoids and Vitamin $\mathrm{E}$ on cyclooxygenase-2 (COX-2) transcription. Mutation Research/Fundamental and Molecular Mechanisms of Mutagenesis. 2004, 551(1-2):245-54

\section{How to cite this article:}

Mahmoud Hashemzaei A, Ameneh Badrooze B, Kaveh Tabrizian C, Mehrdad Iranshahi D, Mehdi Khoshsima Shahraki E, Esmaeil Azimi Khatibani F and Jafar Shahraki G. Effects of Luteolin on intra peritoneal adhesion bands in rat. J. Fundam. Appl. Sci., 2016, 8(2S), 1-11. 